\section{Rückgang des Zervixkarzinoms braucht Zeit}

Friese: Natürlich als Erstes einen deutlichen Rückgang der Dysplasien und der entsprechenden operativen Eingriffe wie Konisationen, was auch zu einer Reduktion der Frühgeburtlichkeit bei den betroffenen Frauen führen wird. In zweiter Linie wird dann als Folge die Inzidenz des Zervixkarzinoms abnehmen. Bis es so weit ist, wird es allerdings eine Weile dauern, wir sprechen hier von Zeitintervallen bis zu 20 Jahren. Wenn ich sehe, wie viele Zervixkarzinome wir allein in München auch bei sehr jungen Patientinnen operieren müssen, dann wäre es sehr gut, wenn man diese Situation mit der Impfung verbessern könnte.

MMW: Dies wäre ja eine regelrechte Revolution in der Medizin.

Friese: Selbstverständlich. Die HPV-Impfung ist ein echter Meilenstein: Wir haben zum ersten Mal in der Geschichte die Option für eine Prävention eines Karzinoms. Durch die Impfung gegen Hepatitis B lässt sich letztlich zwar auch ein Leberzellkarzinom verhindern, doch die eigentliche Intention dieser Impfung ist es, eine Hepatitisinfektion zu vermeiden.

MMW: Die STIKO empfiehlt, Mädchen zwischen 12 und 17 Jahren zu impfen. Ist dies, abgesehen von der großen Altersspanne, nicht ein recht später Zeitpunkt? Friese: Das ist richtig. Um wirklich effektiv zu sein, müssen wir die Mädchen impfen, bevor sie ihren ersten Sexualkontakt haben. Wenn man die Daten aus Europa betrachtet, sieht man, dass nach den Engländerinnen deutsche Mädchen am frühesten Sexualkontakte aufnehmen. Unter den 15-Jährigen hat ein nicht unerheblicher Teil bereits sexuelle Erfahrungen gemacht. Ich denke jedoch, dass wir derzeit keine bessere Lösung finden. Man könnte zwar darüber nachdenken, früher mit der Impfung zu beginnen. Derzeit ist aber noch nicht ganz klar, wie lange die Impfung überhaupt anhält.

\section{Welcher Impfstoff?}

MMW: Beide Impfstoffe, Cervarix ${ }^{\circledR}$ und Gardasil $^{\circledR}$, decken die HPV-Hochrisikotypen 16 und $18 \mathrm{ab}$, Gardasil ${ }^{\circledR}$ zusätzlich die Typen 6 und 11, die Kondylome verursachen. Hat einer der Impfstoffe Vorteile? Friese: So wie es momentan aussieht, schneidet Cervarix ${ }^{\circledR}$ vermutlich aufgrund seines Adjuvans besser ab bezogen auf die immunologischen Daten, aber nicht auf die Effektivität. Jedoch weist Cervarix ${ }^{\circledR}$ eine bessere Kreuzprotektion auf. Ich kann nicht sagen, dass ich derzeit einen der beiden Impfstoffe favorisiere. Das Problem ist, dass sich anfangs viele für den tetravalenten Impfstoff entschieden haben, die Untersuchungen nun aber bei der bivalenten Vakzine auf bessere Ergebnisse hindeuten. Die Entscheidung sollte daher individuell erfolgen.

MMW: Welchen Einfluss würde eine flächendeckende HPV-Impfung auf den Stellenwert der Früherkennungsuntersuchung haben?

Friese: Die Impfung wird nicht die Früherkennungsuntersuchungen ersetzen. Möglicherweise wird es andere Kontrollintervalle geben, aber es ist noch zu früh, um dies sagen zu können. Zum momentanen Zeitpunkt bedeutet dies, dass wir die Früherkennungsuntersuchungen in keinster Weise verändern.

MMW: Soll man nur Mädchen impfen oder auch Jungen?

Friese: Es ist klar, dass unser Impfprogramm viel früher greifen würde, wenn wir auch die Jungen impfen würden. Kosten-Nutzen-Analysen zeigen aber, dass der potenzielle Nutzen zu gering ist, als dass er eine HPV-Impfung von Jungen rechtfertigen würde.

- Interview: Dr. Johannes Weiß Langfassung: Gynäkologe 2009;42:965-68

\title{
Aus für Sibutramin
}

- Der Schlankmacher Sibutramin (Handelsname Reductil ${ }^{\circledR}$ ) ist vom Markt. Am 21. Januar hat die Europäische Arzneimittelagentur (EMA), die Empfehlung ausgesprochen, die Zulassung sibutraminhaltiger Arzneimittel europaweit ruhen zu lassen. Das BfArM (Bundesinstitut für Arzneimittel und Medizinprodukte) reagierte prompt und setzte die Zulassung für Deutschland aus. Damit darf das Anorektikum nicht mehr verschrieben werden; in Apotheken ist das Präparat bereits nicht mehr erhältlich.

Den Stein ins Rollen brachte die 2002 aufgelegte SCOUT-Studie (Sibutramine Cardiovascular Outcome), mit der man den immer wieder aufgetretenen kardiovaskulären Zwischenfällen unter Sibutramineinnahme auf den Grund gehen wollte (in Italien war es deswegen bereits 2002 zu einer vorübergehenden Marktrücknahme gekommen). Konkret sollte SCOUT klären, ob die Gewichtsreduktion mithilfe von Sibutramin bei übergewichtigen/adipösen Hochrisikopatienten Einfluss auf kardiovaskuläre Endpunkte hat.

Im Rahmen der randomisierten doppelblinden placebokontrollierten Studie wurden rund 10000 Patienten über sechs Jahre beobachtet, der primäre Endpunkt setzte sich zusammen aus Herzinfarkt,
Schlaganfall, Wiederbelebung nach Herzstillstand und kardiovaskulärem Tod. Die Ergebnisse liegen seit November 2009 vor, sind aber noch nicht publiziert. Sie zeigen in der Formulierung des BfArM, „dass für übergewichtige Patienten mit zusätzlichen Risikofaktoren die Einnahme von Sibutramin mit einem erhöhten Risiko für das Auftreten schwerwiegender kardiovaskulärer Ereignisse wie z.B. Herzinfarkt oder Schlaganfall verbunden ist." Die von den SCOUT-Patienten erzielte Gewichtsabnahme war zudem nur gering und konnte nicht dazu beitragen, das Herzkreislaufrisiko zu reduzieren.
EO = 